\title{
Principales resultados de la estadística sobre mortalidad en México, 1999
}

$C$ omo ha ocurrido en años resentan seis cuadros que resumen algunos de los datos más relevantes sobre la mortalidad registrada en 1999. Este es el segundo año de uso, en nuestro país, de la nueva Clasificación Internacional de Enfermedades (CIE-10), lo cual permite efectuar comparaciones más directas con el comportamiento de la mortalidad durante el año anterior. Los datos fueron obtenidos mediante el procesamiento de las bases de datos proporcionados a la Secretaría de Salud por el Instituto Nacional de Estadística, Geografía e Informática (INEGI).

No se debe olvidar que el cambio de la CIE-9 a la CIE-10 trajo consigo algunas repercusiones tanto en el número de patologías como en los criterios de selección de la causa básica de defunción. Se espera que tan sólo el efecto del cambio en la clasificación se refleje en un descenso importante de las enfermedades respiratorias tales como neumonía y bronconeumonía y que se eleven las enfermedades infecciosas y parasitarias por la transferencia del síndrome de inmunodeficiencia ad- quirida a ese capítulo. El paro cardíaco desaparece de las enfermedades cardiovasculares puesto que, tratándose de una forma de morir, no constituye realmente una causa, y ahora se codifica dentro del capítulo de signos y síntomas mal definidos.

En ese año destaca una pequeña reducción en el número absoluto de muertes, que contrasta con la tendencia ascendente de los últimos años; esta reducción se traduce en un descenso en las tasas de mortalidad general y para casi todos los grupos de edad. Persisten, como en otros años, los problemas de subregistro de defunciones, particularmente críticos en los estados de Durango, Guerrero, Nayarit y Sinaloa, que hacen imposible un análisis del comportamiento de la mortalidad en esas entidades federativas.

Se aprecia también el avance en el proceso de transición epidemiológica, puesto que continúa el descenso de la mortalidad por enfermedades transmisibles y el ascenso en la proporción de defunciones por enfermedades crónico-degenerativas. Persisten las enfermedades del corazón, los tumores malignos, la diabetes mellitus, los accidentes y las enfermedades del hígado, en ese orden, como las cinco principales causas de muerte en el país; de ellas destaca la diabetes mellitus por su mayor incremento respecto a las demás. Por otra parte, también se reduce el peso relativo de las muertes de los menores de cinco años y de otros grupos de edad antes de los 45 años, mientras que a partir de esa edad se eleva el número de muertes respecto al año anterior. No se aprecian cambios significativos entre los sexos. Estos cambios se pueden observar al comparar cada uno de los seis cuadros con los datos publicados el año anterior.

La Dirección General de Estadística e Informática de la Secretaría de Salud pone a disposición de los lectores el anuario correspondiente, para ampliar la información con relación a otros aspectos de la mortalidad cuya fuente es el certificado de defunción y en donde se pueden apreciar cuadros con datos acerca del nivel educativo, condición laboral, atención médica previa a la muerte, tamaño de la localidad, certificación médica, causas específicas por edad y sexo, etcétera. 


\section{Mortalidad general por seXo, según entidad federativa de residencia habitual. México, 1999}

\begin{tabular}{|c|c|c|c|c|c|c|c|c|}
\hline \multirow[b]{2}{*}{ Entidad federativa } & \multicolumn{2}{|c|}{ Total } & \multicolumn{2}{|c|}{ Hombres } & \multicolumn{2}{|c|}{ Mujeres } & \multirow[b]{2}{*}{$N E$} & \multirow[b]{2}{*}{$I S M$} \\
\hline & Defunciones & $\overline{T a s a}$ & Defunciones & Tasa & Defunciones & Tasa & & \\
\hline Estados Unidos Mexicanos & 443950 & 4.5 & 247833 & 5.1 & 195979 & 4.0 & 2311 & 126.5 \\
\hline Aguascalientes & 3917 & 4.1 & 2135 & 4.5 & 1780 & 3.7 & 19 & 119.9 \\
\hline Baja California & 11301 & 4.9 & 7037 & 6.0 & 4260 & 3.7 & 97 & 165.2 \\
\hline Baja California Sur & 1665 & 4.2 & 993 & 4.9 & 672 & 3.4 & 16 & 147.8 \\
\hline Campeche & 2544 & 3.7 & 1510 & 4.3 & 1033 & 3.0 & 42 & 146.2 \\
\hline Coahuila & 9706 & 4.1 & 5404 & 4.6 & 4301 & 3.7 & 19 & 125.6 \\
\hline Colima & 2351 & 4.4 & 1342 & 5.1 & 1008 & 3.8 & 32 & 133.1 \\
\hline Chiapas & 15204 & 3.8 & 8643 & 4.3 & 6557 & 3.3 & 120 & 131.8 \\
\hline Chihuahua & 15517 & 5.1 & 9013 & 6.0 & 6501 & 4.3 & 100 & 138.6 \\
\hline Distrito Federal & 46601 & 5.3 & 24103 & 5.7 & 22486 & 5.0 & 17 & 107.2 \\
\hline Durango & 6004 & 4.0 & 3485 & 4.6 & 2516 & 3.3 & 28 & 138.5 \\
\hline Guanajuato & 21753 & 4.5 & 11900 & 5.1 & 9851 & 4.0 & 168 & 120.8 \\
\hline Guerrero & 10693 & 3.4 & 6264 & 4.0 & 4423 & 2.8 & 69 & 141.6 \\
\hline Hidalgo & 10038 & 4.4 & 5568 & 4.9 & 4469 & 3.9 & 87 & 124.6 \\
\hline Jalisco & 31300 & 4.9 & 17015 & 5.4 & 14279 & 4.4 & 144 & 119.2 \\
\hline México & 52594 & 4.1 & 29327 & 4.6 & 23238 & 3.6 & 117 & 126.2 \\
\hline Michoacán & 18477 & 4.4 & 10247 & 5.0 & 8222 & 3.9 & 82 & 124.6 \\
\hline Morelos & 6828 & 4.4 & 3772 & 4.9 & 3050 & 3.8 & 73 & 123.7 \\
\hline $\mathrm{N}$ ayarit & 4098 & 4.3 & 2357 & 5.0 & 1739 & 3.7 & 17 & 135.5 \\
\hline Nuevo León & 15836 & 4.2 & 8730 & 4.6 & 7098 & 3.7 & 101 & 123.0 \\
\hline 0 axaca & 18074 & 5.1 & 10115 & 5.8 & 7958 & 4.4 & 121 & 127.1 \\
\hline Puebla & 27169 & 5.4 & 14870 & 6.0 & 12291 & 4.8 & 92 & 121.0 \\
\hline Q uerétaro & 5867 & 4.2 & 3255 & 4.7 & 2605 & 3.7 & 66 & 125.0 \\
\hline Q uintana Roo & 2351 & 3.0 & 1489 & 3.7 & 861 & 2.2 & 67 & 172.9 \\
\hline San Luis Potosí & 10324 & 4.3 & 5687 & 4.8 & 4636 & 3.9 & 31 & 122.7 \\
\hline Sinaloa & 10270 & 4.1 & 6095 & 4.9 & 4172 & 3.4 & 38 & 146.1 \\
\hline Sonora & 10730 & 4.8 & 6396 & 5.7 & 4334 & 4.0 & 33 & 147.6 \\
\hline Tabasco & 7270 & 3.8 & 4328 & 4.5 & 2942 & 3.1 & 28 & 147.1 \\
\hline Tamaulipas & 11563 & 4.3 & 6471 & 4.8 & 5082 & 3.8 & 78 & 127.3 \\
\hline Tlaxcala & 4550 & 4.7 & 2476 & 5.1 & 2070 & 4.2 & 28 & 119.6 \\
\hline Veracruz & 32722 & 4.6 & 18297 & 5.2 & 14424 & 4.1 & 173 & 126.9 \\
\hline Yucatán & 8007 & 4.8 & 4387 & 5.3 & 3619 & 4.4 & 152 & 121.2 \\
\hline Zacatecas & 6323 & 4.4 & 3506 & 5.0 & 2815 & 3.9 & 46 & 124.5 \\
\hline Extranjero & 2303 & & 1616 & & 687 & & 10 & 235.2 \\
\hline
\end{tabular}

Tasa por 1000 habitantes según sexo

Fuente: Instituto N acional de Estadística, Geografía e Informática y Dirección General de Estadística e Informática. Secretaría de Salud, México $N$ E: no especificado

ISM: índice de sobremortalidad masculina 


\section{Cuadro II}

\section{Mortalidad por Grupos de edAd, SEgún entidad federativa de Residencia habitual. MéXico, 1999}

\begin{tabular}{|c|c|c|c|c|c|c|c|c|c|c|c|}
\hline Entidad federativa & Total & $-/$ año & $1-4$ & $5-14$ & $15-24$ & $25-34$ & $35-44$ & $45-54$ & $55-64$ & $65 y^{+}$ & $N E$ \\
\hline Estados Unidos Mexicanos & 443950 & 40283 & 7774 & 7483 & 18191 & 23670 & 29783 & 39434 & 56027 & 218994 & 2311 \\
\hline Aguascalientes & 3917 & 412 & 71 & 72 & 146 & 182 & 207 & 333 & 464 & 2011 & 19 \\
\hline Baja California & 11301 & 1169 & 185 & 176 & 588 & 999 & 1050 & 1186 & 1376 & 4475 & 97 \\
\hline Baja California Sur & 1665 & 149 & 24 & 20 & 70 & 102 & 121 & 160 & 222 & 781 & 16 \\
\hline Campeche & 2544 & 210 & 47 & 37 & 125 & 127 & 179 & 245 & 327 & 1205 & 42 \\
\hline Coahuila & 9706 & 521 & 86 & 115 & 347 & 436 & 539 & 914 & 1610 & 5119 & 19 \\
\hline Colima & 2351 & 158 & 37 & 27 & 93 & 108 & 136 & 192 & 297 & 1271 & 32 \\
\hline Chiapas & 15204 & 1539 & 536 & 467 & 997 & 1155 & 1357 & 1481 & 1749 & 5803 & 120 \\
\hline Chihuahua & 15517 & 1284 & 223 & 235 & 726 & 943 & 1095 & 1433 & 2163 & 7315 & 100 \\
\hline Distrito Federal & 46601 & 3323 & 381 & 376 & 1326 & 2125 & 2932 & 4173 & 6155 & 25793 & 17 \\
\hline Durango & 6004 & 149 & 46 & 82 & 298 & 307 & 378 & 486 & 811 & 3419 & 28 \\
\hline Guanajuato & 21753 & 2577 & 460 & 427 & 801 & 990 & 1175 & 1704 & 2293 & 11158 & 168 \\
\hline Guerrero & 10693 & 807 & 256 & 218 & 589 & 793 & 887 & 973 & 1364 & 4737 & 69 \\
\hline Hidalgo & 10038 & 899 & 167 & 177 & 419 & 451 & 705 & 976 & 1239 & 4918 & 87 \\
\hline Jalisco & 31300 & 2440 & 436 & 523 & 1126 & 1427 & 1815 & 2491 & 3589 & 17309 & 144 \\
\hline México & 52594 & 7531 & 958 & 898 & 2376 & 3107 & 3908 & 5357 & 6894 & 21448 & 117 \\
\hline Michoacán & 18477 & 1233 & 265 & 351 & 834 & 994 & 1184 & 1514 & 2097 & 9923 & 82 \\
\hline Morelos & 6828 & 477 & 104 & 88 & 271 & 353 & 496 & 577 & 856 & 3533 & 73 \\
\hline $\mathrm{N}$ ayarit & 4098 & 187 & 59 & 78 & 149 & 213 & 233 & 296 & 510 & 2356 & 17 \\
\hline Nuevo León & 15836 & 1024 & 148 & 165 & 510 & 661 & 911 & 1353 & 2273 & 8690 & 101 \\
\hline 0 axaca & 18074 & 1453 & 555 & 417 & 793 & 992 & 1224 & 1567 & 2166 & 8786 & 121 \\
\hline Puebla & 27169 & 3893 & 793 & 615 & 992 & 1243 & 1827 & 2170 & 3113 & 12431 & 92 \\
\hline Q uerétaro & 5867 & 748 & 117 & 114 & 227 & 291 & 429 & 538 & 671 & 2666 & 66 \\
\hline Q uintana Roo & 2351 & 333 & 48 & 52 & 158 & 217 & 210 & 250 & 264 & 752 & 67 \\
\hline San Luis Potosí & 10324 & 906 & 198 & 190 & 367 & 476 & 519 & 804 & 1173 & 5660 & 31 \\
\hline Sinaloa & 10270 & 328 & 147 & 181 & 496 & 585 & 628 & 858 & 1299 & 5710 & 38 \\
\hline Sonora & 10730 & 743 & 158 & 153 & 422 & 573 & 710 & 944 & 1554 & 5440 & 33 \\
\hline Tabasco & 7270 & 773 & 167 & 193 & 415 & 528 & 544 & 703 & 837 & 3082 & 28 \\
\hline Tamaulipas & 11563 & 690 & 148 & 148 & 464 & 571 & 733 & 1048 & 1750 & 5933 & 78 \\
\hline Tlaxcala & 4550 & 705 & 97 & 73 & 154 & 195 & 283 & 344 & 475 & 2196 & 28 \\
\hline Veracruz & 32722 & 2532 & 582 & 551 & 1171 & 1679 & 2355 & 3086 & 4578 & 16015 & 173 \\
\hline Yucatán & 8007 & 589 & 124 & 90 & 226 & 271 & 409 & 567 & 871 & 4708 & 152 \\
\hline Zacatecas & 6323 & 479 & 105 & 122 & 256 & 261 & 296 & 411 & 680 & 3667 & 46 \\
\hline Extranjero & 2303 & 22 & 46 & 52 & 259 & 315 & 308 & 300 & 307 & 684 & 10 \\
\hline
\end{tabular}

Fuente: Instituto N acional de Estadística, Geografía e Informática y Dirección General de Estadística e Informática. Secretaría de Salud, México $N E$ : no especificado 


\section{Mortalidad POR enfermedades transmisibles, NO tRANSMISIBLES Y Lesiones. México, 1999}

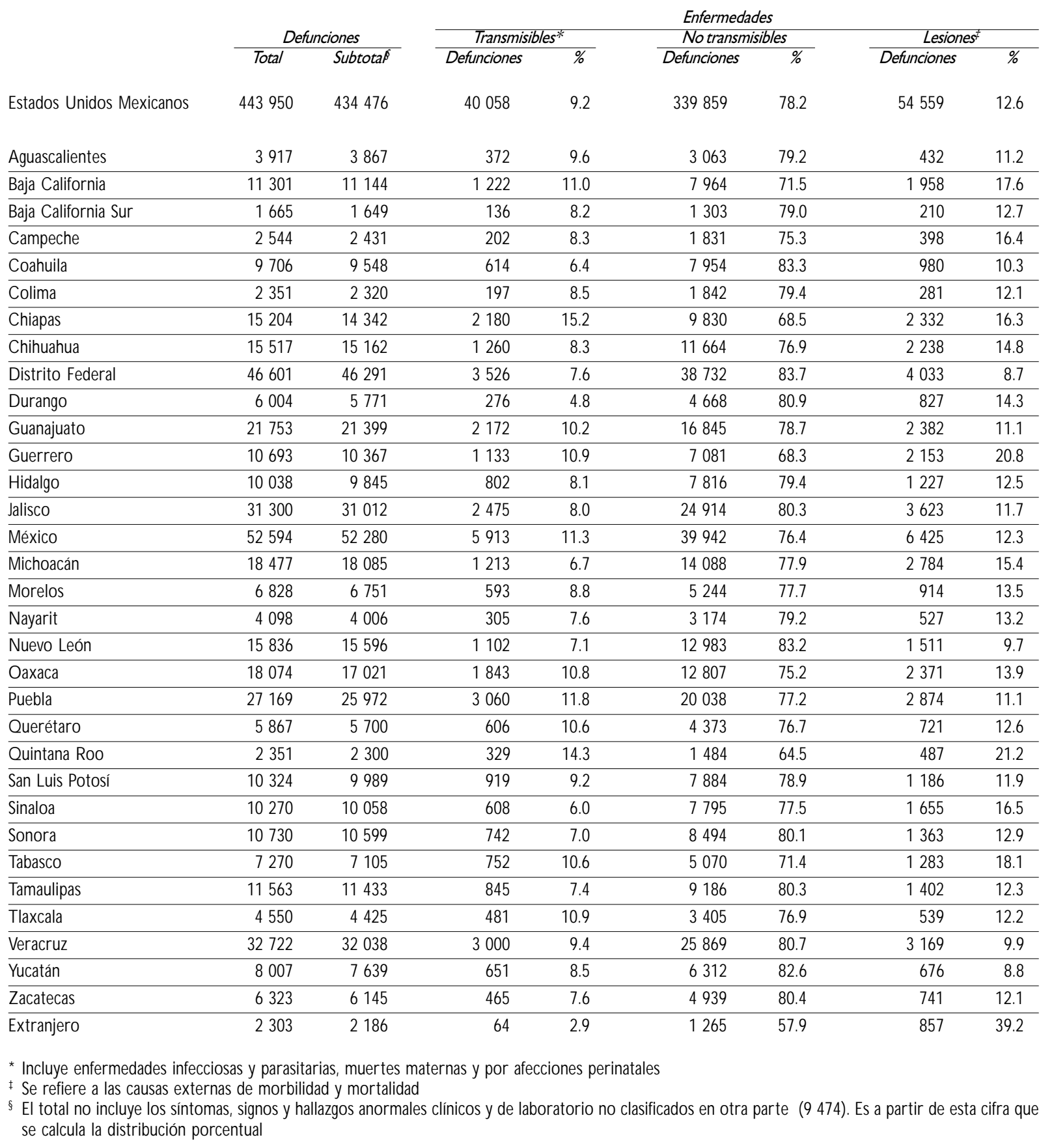

Fuente: Instituto N acional de Estadística Geografía e Informática y Dirección General de Estadística e Informática. Secretaría de Salud, México 


\section{Cuadro IV \\ Principales causas de mortalidad general. México, 1999}

\begin{tabular}{|c|c|c|c|c|c|}
\hline \multirow[t]{2}{*}{ Número de orden } & Criterio de agrupación & Causa & Defunciones & Tasa* & $\%$ \\
\hline & 000 & Total & 443950 & 452.4 & 100.0 \\
\hline \multirow[t]{2}{*}{1} & 097 & Enfermedades del corazón & 69278 & 70.6 & 15.6 \\
\hline & 097C & - Enfermedades isquémicas del corazón & 44070 & 44.9 & 9.9 \\
\hline 2 & 049 & Tumores malignos & 53662 & 54.7 & 12.1 \\
\hline 3 & 061 & Diabetes mellitus & 45632 & 46.5 & 10.3 \\
\hline \multirow[t]{2}{*}{4} & 167 & Accidentes & 35690 & 36.4 & 8.0 \\
\hline & 167B & - Accidentes de tráfico de vehículos de motor & 11659 & 11.9 & 2.6 \\
\hline \multirow[t]{2}{*}{5} & 125 & Enfermedades del hígado & 27040 & 27.6 & 6.1 \\
\hline & $125 \mathrm{~A}$ & - Enfermedad alcohólica del hígado & 13417 & 13.7 & 3.0 \\
\hline 6 & 098 & Enfermedades cerebrovasculares & 25836 & 26.3 & 5.8 \\
\hline \multirow[t]{2}{*}{7} & 163 & Ciertas afecciones originadas en el periodo perinatal & 19268 & 19.6 & 4.3 \\
\hline & $163 \mid$ & $\begin{array}{l}\text { Dificultad respiratoria del recién nacido y otros trastornos } \\
\text { respiratorios originados en el periodo perinatal }\end{array}$ & 10042 & 10.2 & 2.3 \\
\hline 8 & 105 & Influenza y neumonía & 14068 & 14.3 & 3.2 \\
\hline 9 & 169 & Agresiones (homicidio) & 12249 & 12.5 & 2.8 \\
\hline 10 & 113 & Enfermedades pulmonares obstructivas crónicas & 11319 & 11.5 & 2.6 \\
\hline 11 & 066 & Desnutrición y otras deficiencias nutricionales & 9776 & 10.0 & 2.2 \\
\hline 12 & 165 & Malformaciones congénitas, deformidades y anomalías cromosómicas & 9714 & 9.9 & 2.2 \\
\hline 13 & 110 & Bronquitis crónica y la no especificada, enfisema y asma & 7840 & 8.0 & 1.8 \\
\hline 14 & 139 & Insuficiencia renal & 7807 & 8.0 & 1.8 \\
\hline 15 & 001 & Enfermedades infecciosas intestinales & 5622 & 5.7 & 1.3 \\
\hline 16 & 037 & Enfermedad por virus de la inmunodeficiencia humana (SIDA) & 4204 & 4.3 & 1.0 \\
\hline 17 & 058 & Anemias & 3581 & 3.7 & 0.8 \\
\hline 18 & 168 & Lesiones autoinfligidas intencionalmente (suicidio) & 3339 & 3.4 & 0.8 \\
\hline 19 & 002 & Tuberculosis pulmonar & 3229 & 3.3 & 0.7 \\
\hline \multirow[t]{5}{*}{20} & 015 & Septicemia & 3085 & 3.1 & 0.7 \\
\hline & 997 & Paro cardíaco & 0 & 0.0 & 0.0 \\
\hline & 998 & Síntomas, signos y hallazgos anormales clínicos y de laboratorio & & & \\
\hline & & no clasificados en otra parte & 9474 & 9.7 & 2.1 \\
\hline & 999 & Las demás causas & 62237 & 63.4 & 14.0 \\
\hline
\end{tabular}

*Tasa por 100000 habitantes estimada con base en las proyecciones de población del Consejo Nacional de Población

Fuente: Instituto N acional de Estadística, Geografía e Informática y Dirección General de Estadística e Informática. Secretaría de Salud, México 


\section{Cuadro V}

\section{Años POTENCIALES DE VIDA PERDIDOS Y EDAD PROMEDIO A LA MUERTE,} SEgún entidad federativa de Residencia habitual. MéXICO, 1999

\begin{tabular}{|c|c|c|c|c|c|c|}
\hline Entidad federativa & Total & Tasa* & Edad promedio a la muerte & Años de vida potencial perdidost & Tasa AVPPs & $\%$ respecto a AVPP \\
\hline Estados Unidos Mexicanos & 443950 & 4.5 & 56.7 & 7916130.5 & 80.7 & 100.0 \\
\hline Aguascalientes & 3917 & 4.1 & 57.1 & 69977.0 & 72.6 & 0.9 \\
\hline Baja California & 11301 & 4.9 & 51.2 & 243978.0 & 104.7 & 3.1 \\
\hline Baja California Sur & 1665 & 4.2 & 56.0 & 29849.5 & 74.8 & 0.4 \\
\hline Campeche & 2544 & 3.7 & 56.2 & 45082.5 & 64.7 & 0.6 \\
\hline Coahuila & 9706 & 4.1 & 60.5 & 135060.5 & 57.4 & 1.7 \\
\hline Colima & 2351 & 4.4 & 60.3 & 35181.5 & 66.0 & 0.4 \\
\hline Chiapas & 15204 & 3.8 & 49.8 & 356531.0 & 89.3 & 4.5 \\
\hline Chihuahua & 15517 & 5.1 & 55.9 & 277736.5 & 92.2 & 3.5 \\
\hline Distrito Federal & 46601 & 5.3 & 60.7 & 668298.0 & 76.2 & 8.4 \\
\hline Durango & 6004 & 4.0 & 62.4 & 75318.5 & 49.6 & 1.0 \\
\hline Guanajuato & 21753 & 4.5 & 56.6 & 409482.5 & 85.0 & 5.2 \\
\hline Guerrero & 10693 & 3.4 & 54.3 & 208869.5 & 66.7 & 2.6 \\
\hline Hidalgo & 10038 & 4.4 & 57.1 & 177187.5 & 77.7 & 2.2 \\
\hline Jalisco & 31300 & 4.9 & 60.0 & 486387.5 & 75.7 & 6.1 \\
\hline México & 52594 & 4.1 & 50.9 & 1183309.0 & 92.2 & 14.9 \\
\hline Michoacán & 18477 & 4.4 & 59.5 & 294858.0 & 70.5 & 3.7 \\
\hline Morelos & 6828 & 4.4 & 58.9 & 108979.5 & 69.9 & 1.4 \\
\hline $\mathrm{N}$ ayarit & 4098 & 4.3 & 61.9 & 56130.5 & 59.1 & 0.7 \\
\hline Nuevo León & 15836 & 4.2 & 60.8 & 221707.0 & 58.2 & 2.8 \\
\hline 0 axaca & 18074 & 5.1 & 56.2 & 334267.5 & 94.3 & 4.2 \\
\hline Puebla & 27169 & 5.4 & 52.7 & 592405.0 & 117.1 & 7.5 \\
\hline Q uerétaro & 5867 & 4.2 & 53.6 & 120961.0 & 87.4 & 1.5 \\
\hline Q uintana Roo & 2351 & 3.0 & 46.1 & 60327.5 & 76.0 & 0.8 \\
\hline San Luis Potosí & 10324 & 4.3 & 59.2 & 169577.5 & 71.2 & 2.1 \\
\hline Sinaloa & 10270 & 4.1 & 61.3 & 141961.5 & 57.1 & 1.8 \\
\hline Sonora & 10730 & 4.8 & 58.0 & 172546.0 & 77.9 & 2.2 \\
\hline Tabasco & 7270 & 3.8 & 52.2 & 157666.0 & 82.8 & 2.0 \\
\hline Tamaulipas & 11563 & 4.3 & 59.2 & 174283.5 & 64.6 & 2.2 \\
\hline Tlaxcala & 4550 & 4.7 & 54.0 & 95829.5 & 98.7 & 1.2 \\
\hline Veracruz & 32722 & 4.6 & 57.4 & 555231.5 & 78.7 & 7.0 \\
\hline Yucatán & 8007 & 4.8 & 61.9 & 109864.0 & 66.5 & 1.4 \\
\hline Zacatecas & 6323 & 4.4 & 61.1 & 95423.5 & 66.7 & 1.2 \\
\hline
\end{tabular}

* Tasa por 1000 habitantes

₹ Es la suma de los años de vida potencial perdidos por cada una de las defunciones ocurridas, respecto a un límite superior de la edad (70.0)

$\S$ Tasa de años de vida potencial perdidos por 1000 habitantes, estimada con base en las proyecciones de población del Consejo Nacional de Población

Fuente: Instituto N acional de Estadística, Geografía e Informática y Dirección General de Estadística e Informática. Secretaría de Salud, México 


\section{Cuadro VI \\ MORTALIDAD POR ENFERMEDADES INFECCIOSAS INTESTINALES, INFECCIONES RESPIRATORIAS AGUDAS Y SÍNDROME DE INMUNODEFICIENCIA ADQUIRIDA, SEgún entidad federativa de ReSidencia habitual. MéXICO, 1999}

\begin{tabular}{|c|c|c|c|c|c|c|}
\hline \multirow[b]{2}{*}{ Entidad federativa } & \multicolumn{2}{|c|}{$\begin{array}{c}\text { Enfermedades infecciosas } \\
\text { intestinales }\end{array}$} & \multicolumn{2}{|c|}{$\begin{array}{l}\text { Infecciones respiratorias } \\
\text { agudas }\end{array}$} & \multicolumn{2}{|c|}{$\begin{array}{l}\text { Enfermedad por virus de la } \\
\text { inmunodeficiencia humana (SIDA) }\end{array}$} \\
\hline & Defunciones & Tasa* & Defuncines & Tasa* & Defunciones & Tasa* \\
\hline Estados Unidos Mexicanos & 5622 & 5.7 & 16674 & 17.0 & 4204 & 4.3 \\
\hline Aguascalientes & 36 & 3.7 & 76 & 7.9 & 26 & 2.7 \\
\hline Baja California & 70 & 3.0 & 388 & 16.7 & 234 & 10.0 \\
\hline Baja California Sur & 13 & 3.3 & 46 & 11.5 & 15 & 3.8 \\
\hline Campeche & 19 & 2.7 & 69 & 9.9 & 20 & 2.9 \\
\hline Coahuila & 54 & 2.3 & 166 & 7.1 & 48 & 2.0 \\
\hline Colima & 23 & 4.3 & 54 & 10.1 & 33 & 6.2 \\
\hline Chiapas & 597 & 14.9 & 725 & 18.1 & 115 & 2.9 \\
\hline Chihuahua & 149 & 4.9 & 538 & 17.9 & 112 & 3.7 \\
\hline Distrito Federal & 328 & 3.7 & 1813 & 20.7 & 689 & 7.9 \\
\hline Durango & 35 & 2.3 & 147 & 9.7 & 38 & 2.5 \\
\hline Guanajuato & 391 & 8.1 & 846 & 17.6 & 113 & 2.3 \\
\hline Guerrero & 202 & 6.4 & 314 & 10.0 & 145 & 4.6 \\
\hline Hidalgo & 98 & 4.3 & 380 & 16.7 & 37 & 1.6 \\
\hline Jalisco & 256 & 4.0 & 1160 & 18.1 & 394 & 6.1 \\
\hline México & 831 & 6.5 & 3120 & 24.3 & 481 & 3.7 \\
\hline Michoacán & 184 & 4.4 & 564 & 13.5 & 103 & 2.5 \\
\hline Morelos & 80 & 5.1 & 186 & 11.9 & 116 & 7.4 \\
\hline $\bar{N}$ ayarit & 36 & 3.8 & 126 & 13.3 & 40 & 4.2 \\
\hline Nuevo León & 69 & 1.8 & 413 & 10.8 & 154 & 4.0 \\
\hline 0 axaca & 516 & 14.6 & 828 & 23.4 & 113 & 3.2 \\
\hline Puebla & 467 & 9.2 & 1499 & 29.6 & 211 & 4.2 \\
\hline Q uerétaro & 81 & 5.8 & 156 & 11.3 & 26 & 1.9 \\
\hline Q uintana Roo & 27 & 3.4 & 49 & 6.2 & 52 & 6.6 \\
\hline San Luis Potosí & 143 & 6.0 & 370 & 15.5 & 54 & 2.3 \\
\hline Sinaloa & 80 & 3.2 & 250 & 10.0 & 75 & 3.0 \\
\hline Sonora & 98 & 4.4 & 255 & 11.5 & 46 & 2.1 \\
\hline Tabasco & 58 & 3.0 & 183 & 9.6 & 87 & 4.6 \\
\hline Tamaulipas & 58 & 2.1 & 224 & 8.3 & 79 & 2.9 \\
\hline Tlaxcala & 69 & 7.1 & 257 & 26.5 & 26 & 2.7 \\
\hline Veracruz & 365 & 5.2 & 974 & 13.8 & 395 & 5.6 \\
\hline Yucatán & 101 & 6.1 & 242 & 14.6 & 82 & 5.0 \\
\hline Zacatecas & 84 & 5.9 & 211 & 14.7 & 22 & 1.5 \\
\hline Extranjero & 4 & & 45 & & 23 & \\
\hline
\end{tabular}

* Tasa por 100000 habitantes con base en las proyecciones de población del Consejo Nacional de Población

Fuente: Instituto N acional de Estadística, Geografía e Informática y Dirección General de Estadística e Informática. Secretaría de Salud, México 